\title{
Laparoscopic radical prostatectomy and extended pelvic lymph node dissection: a combined technique
}

\author{
Piotr Jarzemski ${ }^{1}$, Sławomir Listopadzki ${ }^{1}$, Piotr Słupski ${ }^{1}$, Marcin Jarzemski ${ }^{1}$, Bartosz Brzoszczyk ${ }^{1}$, Roman Sosnowski ${ }^{2}$ \\ ${ }^{1}$ Department of Urology, Jan Biziel University Hospital in Bydgoszcz, Nicolaus Copernicus University in Torun, Poland \\ ${ }^{2}$ Department of Urooncology, M. Sklodowska-Curie Memorial Cancer Center and Institute of Oncology, Warsaw, Poland
}

Videosurgery Miniinv 2020; 15 (1): 192-198

DOI: https://doi.org/10.5114/wiitm.2019.86810

\begin{abstract}
Introduction: The important part of radical prostatectomy $(R P)$ for high risk $(H R)$ is extended pelvic lymph node dissection (ePLND). This method consists of two stages of surgery usually performed at the compartment (pre-or transperineally).

Aim: We present our new combined technique of RP using two different approaches: a pre-peritoneal approach for laparoscopic radical prostatectomy (LRP) and a transperitoneal approach for ePLND.

Material and methods: This study included 30 patients aged 53 to 75 years (mean age: 64 years) with prostate cancer who underwent LRP and ePLND using a combined technique. After the pre-peritoneal LRP, transposition of the trocars into the peritoneal cavity was performed without changing their location, except the extreme left trocar, which was inserted through a new approach.

Results: The total duration of surgery was 155 to 290 min (mean: 215 min); ePLND lasted from 35 to 85 min (mean: $56 \mathrm{~min}$ ). The movement of trocars into the peritoneal cavity was a very simple maneuver, taking up to 1 min without any complications. The number of removed lymph nodes (LNS) ranged from 13 to 28 (mean: 16.8). A positive margin was found in 5 (16\%) patients. We recognized positive nodes in 9 (30\%) patients.

Conclusions: The combined technique is both feasible and safe. Performing the most difficult maneuver, removal of the prostate, in the first stage appears to be more comfortable for the operator. The timing of the PLND stage in the combined technique and the number of removed $L N$ s do not differ from the standard lenticular access.
\end{abstract}

Key words: laparoscopy, prostate cancer, lymph node dissection.

\section{Introduction}

Prostate cancer is the second most commonly diagnosed malignancy and the fifth leading cause of death from cancer in men worldwide [1]. Despite prostate-specific antigen (PSA)-based screening and early detection guidelines, approximately $15-26 \%$ of prostate cancer patients present with high-risk (HR) features indicative of a more advanced and potentially lethal course [2]. Patients with more advanced or poorly differentiated tumors could also potentially benefit from surgery [3]. Interest in radical prostatectomy (RP) as a treatment option for patients with HR disease has increased over the past decade. This trend stems from growing evidence that surgery, either alone or in combination with adjuvant treatments, is associated with favorable cancer control outcomes $[4,5]$. Intuitively, surgery may be more beneficial when complete removal of the disease is possible, in patients with tumors confined to the RP specimen. This hypothesis is supported by more favorable survival rates in HR prostate cancer patients harboring 
organ-confined disease at the RP [6]. Guidelines from the European Association of Urology (EAU) now support a role of RP in the treatment of select HR patients, which may include a multimodal approach [7]. The goal of surgery in a HR patient is to achieve a good oncological outcome. Surgical treatment includes extended lymph node dissection (ePLND), clean apical dissection, neurovascular bundle resection on the tumor-bearing side, complete resection of the seminal vesicles, or resection of the bladder neck [8]. A radical procedure consists of two operations performed at the same time, involving prostate removal and ePLND. Surgery can be performed by either a transperitoneal or an extraperitoneal approach. However, transperitoneal access seems to be more advantageous because higher rates of complications, such as symptomatic lymphoceles, appear to be attributed to the extraperitoneal approach [8].

\section{Aim}

The aim of the study is to present our new combined technique of RP using two different approaches: a pre-peritoneal approach for laparoscopic radical prostatectomy (LRP) and a transperitoneal approach for ePLND.

\section{Material and methods}

This prospective study included 30 patients aged 53 to 75 years (mean age: 64 years) diagnosed with prostate cancer who qualified for LRP with ePLND by combined technique. We developed a new technique for the extraperitoneal approach to remove the prostate first and a transperitoneal approach for ePLND after prostatectomy. All patients were qualified for invasive treatment according to EAU prostate cancer guidelines: intermediate-risk patients with a pre-operative risk nomogram indicating $>5 \%$ likelihood of lymph node metastasis and all high-risk patients. Every participant was informed about this technique, received an explanation of the details, and provided signed informed consent. All of the procedures were performed by two experienced urologists. We evaluated all histopathological parameters before and after the operation, procedure details, and operator's comments about the combined approach.

Pre-operative prostate volume measured by magnetic resonance imaging ranged from 20 to $122 \mathrm{ml}$ (mean: $47.1 \mathrm{ml}$ ) and PSA values from 4.3 to 54.29 ng/ml (mean: $17.04 \mathrm{ng} / \mathrm{ml}$ ). The clinical stage was
cT2 in 14 patients and cT3 in 16 patients. The degree of malignancy according to the Gleason scale (GS) was dominated by GS $\geq 8$ (23 patients, 77\%; Table I). According to the 2014 classification of the International Society of Urological Pathology (ISUP), ISUP grade $\geq 4$ was predominant (19 patients, 63\%). There were no patients with suspicion of metastasis in MRI or bone scan imaging. Multiparametric magnetic resonance imaging ( $\mathrm{mpMRI}$ ) was used to measure changes on the Prostate Imaging and Reporting and Data System (PIRADSv2) scale; 28 (93\%) patients were PIRADS 4 or 5 . In the mpMRI study,

Table I. Preoperative characteristics of the patients

\begin{tabular}{|c|c|}
\hline Parameter & Number of patients \\
\hline Total & 30 \\
\hline \multicolumn{2}{|l|}{ Clinical stage: } \\
\hline cT2 & 14 \\
\hline cT3 & 16 \\
\hline \multicolumn{2}{|c|}{ Degree of malignancy according to the Gleason scale: } \\
\hline 9 & 5 \\
\hline 8 & 14 \\
\hline 7 & 7 \\
\hline 6 & 4 \\
\hline \multicolumn{2}{|c|}{ Degree of malignancy according to the ISUP: } \\
\hline Grade 5 & 5 \\
\hline Grade 4 & 14 \\
\hline Grade 3 & 3 \\
\hline Grade 2 & 4 \\
\hline Grade 1 & 4 \\
\hline \multicolumn{2}{|l|}{ mpMRI results: } \\
\hline PIRADS 5 & 10 \\
\hline PIRADS 4 & 18 \\
\hline PIRADS 3 & 2 \\
\hline ECE on mpMRI & 13 \\
\hline LNI on mpMRI & 10 \\
\hline \multicolumn{2}{|l|}{ Probability of $\mathrm{N}+$ : } \\
\hline Briganti nomogram > 5\% & 25 \\
\hline Briganti nomogram $<5 \%$ & 5 \\
\hline
\end{tabular}

ECE - extracapsular extension, ISUP - International Society of Urological Pathology, LNI - lymph node invasion, mpMRI - multiparametric magnetic resonance imaging, PIRADS - Prostate Imaging and Reporting and Data System. 
13 patients were suspected of extracapsular extension (ECE) and 10 lymph node invasion (LNI). The probability of lymph node (LN) metastasis according to the Briganti nomogram > 5\% concerned 25 (83\%) patients. ePLND was performed in these patients. For the remaining 5 patients, the decision to execute ePLND was due to suspicion on multi-parametric magnetic resonance imaging (mpMRI) of LNI and/ or ECE (Table I). Each time, the type of procedure was discussed with the patient, who made the final decision and gave written consent for the procedure to be carried out.

\section{Operative technique}

Thirty patients were planned for RP with expanded pelvic lymphadenectomy performed by a standard LRP technique, a combined technique to remove LNs. The operation was performed using both extraperitoneal and transperitoneal approaches simultaneously.

In the first stage, RP was performed using pre-peritoneal access, preserving all the rules of the procedure for HR prostate cancer. Five trocars were used in typical places. After the RP, ePLND was performed according to our own technique. In the first stage, transposition of the trocars was performed. First, a trocar placed under the peritoneum was inserted under the navel for optics. $\mathrm{CO}_{2}$ was administered through this trocar, insufflation performed, and the remaining three trocars trans-

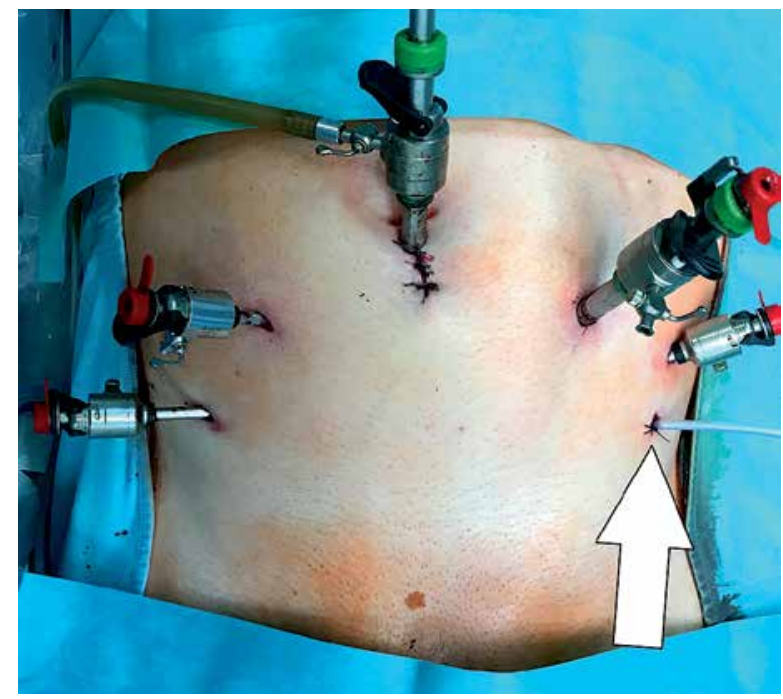

Photo 1. The drain in the pre-peritoneal space remained open during the entire second stage of pelvic LN removal posed into the peritoneal cavity under vision control without changing their location. The exception was the extreme left trocar, which was inserted through the new opening above the drain previously left in the space before retardation. In the pre-peritoneal space, the tube was left in place of the $5 \mathrm{~mm}$ trocar, the one furthest on the left. The drain in the pre-peritoneal space remained open during the entire second stage of pelvic LN removal (Photo 1). Removal of the pelvic LNs was then initiated. First, we identified the iliac vessels and ureter crossing the common iliac artery (Photo 2). All treatments proceeded according to the same scheme, starting from the right. The presacral LNs were always removed first. The procedure began by dissecting the peritoneum medially from the right ureter (Photo 2). The presacral nodes were then removed from the promontory to reveal the median sacral artery (Photo 3). The median sacral artery arises just above the aortic bifurcation into the common iliac arteries. Next, we moved medially from the vein and common iliac artery towards the triangle of Marcille (the lumbosacral triangle) (Photo 4). The tissue was removed from the common iliac artery and the ureter dissected. The proximal extent of dissection was controlled with $5 \mathrm{~mm}$ hem-o-loc clips and divided. The external iliac artery was skeletonized from the origin of the artery and nerve genitofemoralis (Photo 5). The limits of dissection were the common iliac proximally to the inguinal ligament (the node of Cloquet) distally. In the next stage, we proceeded to prepare the obturator hole. The obturator nerve, which is on the floor of this dissection, was carefully preserved. We did not cut the vas deferens. The vasa deferentia were retracted, providing excellent visualization of

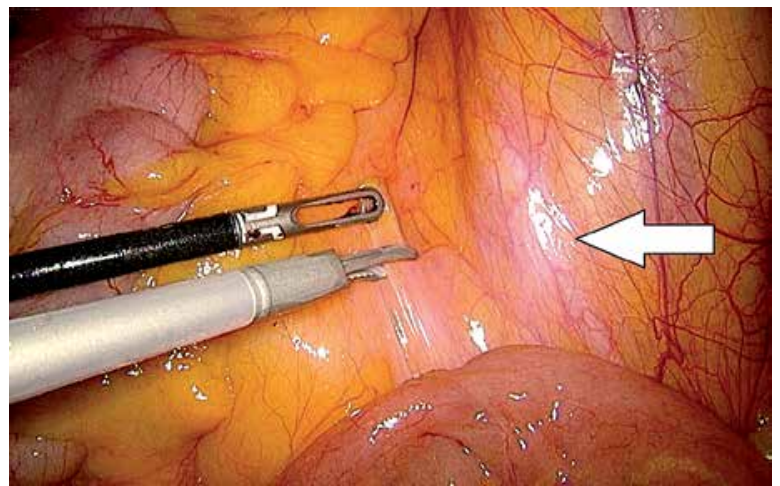

Photo 2. The hip vessels and ureter crossing the common iliac artery 


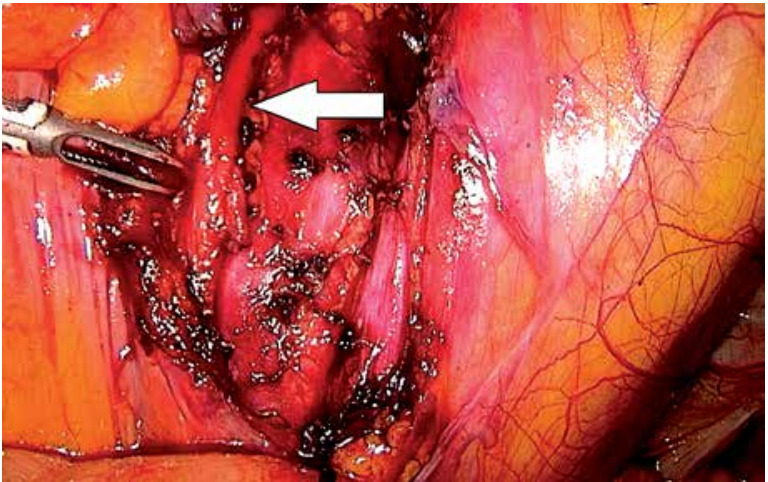

Photo 3. The presacral nodes from the promontory to reveal the median sacral artery

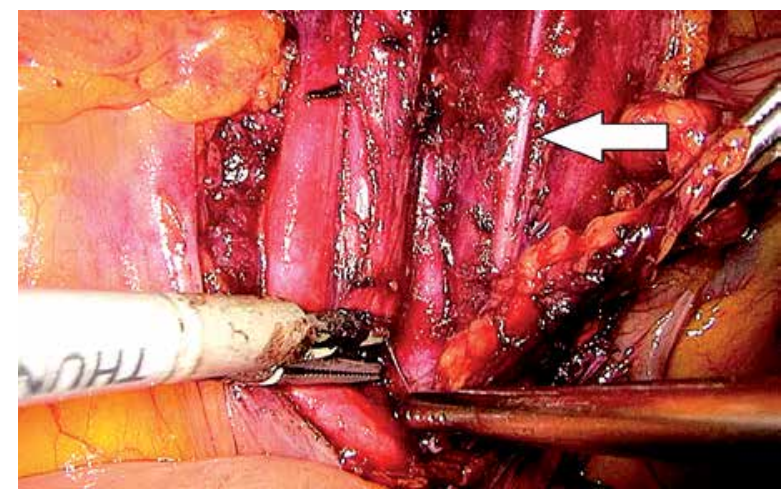

Photo 5. The external iliac artery and genitofemoralis nerve location

the $L N$ packets. At the end of the internal iliac, the group of nodes was removed. Lymphadenopathic tissue surrounding the internal iliacs and branches was also removed. Nodal tissue around the internal iliac often coalesced with the obturator packet anteriorly and posteriorly to the lateral sacral nodes. The limit of the dissection was the lateral sacral arteries arising from the posterior division of the internal iliac vein (Photo 6). The tissues on the left side were removed in the same way and the procedure terminated by leaving the second tube in the vesiculo-rectal recess.

\section{Results}

All 30 patients underwent LRP and ePLND using a combined technique. The results of histopathological examination were as follows: adenocarcinoma of the prostate, stage: T2a -2 , pT2c -8 , pT3a -6 , pT3b-14; for grades of malignancy according to the Gleason scale and ISUP (Table II). A positive margin was found in 5 (16\%) patients, including 3 patients

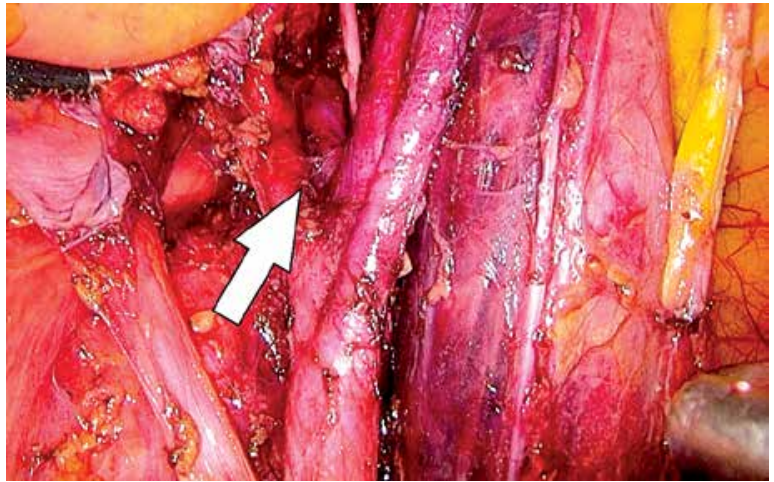

Photo 4. The vein and iliac artery towards the Triangle of Marcille (the lumbosacral triangle)

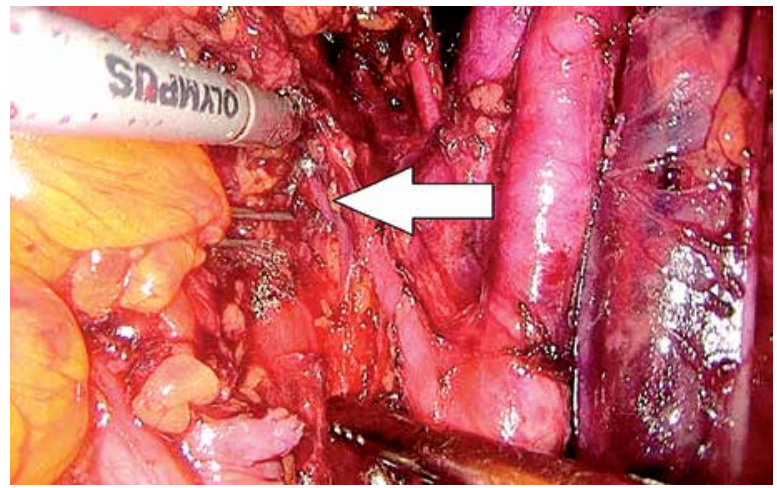

Photo 6. The lateral sacral arteries arising from the posterior division of the internal iliac vessel

out of 13 with extracapsular extension based on mpNMR. The total duration of surgery was 155 to 290 min (mean: $215 \mathrm{~min}$ ); the pelvic lymphadenectomy lasted from 35 to 85 min (mean: 56 min; right side: 20-45 min, mean $30 \mathrm{~min}$; left side: 15-40 min, $26 \mathrm{~min}$ ). The mean blood loss was $180 \mathrm{ml}$ (200$400 \mathrm{ml}$ ). The movement of trocars into the peritoneal cavity was a very simple maneuver, taking up to 1 min without any complications. We recognized positive nodes in 9 (30\%) patients. The number of removed LNs ranged from 13 to 28 (mean: 16.8). The most common location of metastatic lesions was the internal iliac junction (11 nodes), followed by obturator (8 nodes), presacral (5 nodes), and external iliac (4 nodes). The drain from the pre-peritoneal space was removed in the first $24 \mathrm{~h}$. The drain from the peritoneum was removed when lymph leakage fell below $300 \mathrm{ml}$ and the same day patients were discharged home. Six weeks after surgery, PSA was undetectable in 23 (77\%) patients. In 7 patients, the PSA level ranged from 0.09 to $10.0 \mathrm{ng} / \mathrm{ml}$ (3 patients 
Table II. Postoperative characteristics of the patients

\begin{tabular}{|c|c|}
\hline Parameter & Number of patients \\
\hline Total & 30 \\
\hline \multicolumn{2}{|l|}{ Clinical stage: } \\
\hline pT2a & 2 \\
\hline pT2c & 8 \\
\hline рТ3а & 6 \\
\hline pT3b & 14 \\
\hline \multicolumn{2}{|c|}{ Degree of malignancy according to the Gleason scale: } \\
\hline 9 & 10 \\
\hline 8 & 5 \\
\hline 7 & 10 \\
\hline 6 & 5 \\
\hline \multicolumn{2}{|c|}{ Degree of malignancy according to the ISUP: } \\
\hline Grade 5 & 10 \\
\hline Grade 4 & 5 \\
\hline Grade 3 & 2 \\
\hline Grade 2 & 8 \\
\hline Grade 1 & 5 \\
\hline Margin + & 5 \\
\hline Number of patients with LNI: & 9 \\
\hline 1 node $\mathrm{N}+$ & 4 \\
\hline 2 nodes $\mathrm{N}+$ & 1 \\
\hline 5 nodes $\mathrm{N}+$ & 2 \\
\hline 6 nodes $\mathrm{N}+$ & 2 \\
\hline
\end{tabular}

from 0.1 to $0.5 \mathrm{ng} / \mathrm{ml}$, and $4>0.5 \mathrm{ng} / \mathrm{ml})$. $\ln 11(36 \%)$ patients, radiotherapy and/or hormone therapy was recommended.

\section{Complications}

We recorded complications in three patients. One patient suffered bleeding into the peritoneal cavity from the kidney vessels and underwent surgery and blood transfusion. In another patient, a leaky anastomosis was identified. The third patient suffered a subcutaneous pneumothorax, which was treated conservatively.

\section{Discussion}

Though the role and potential benefits of ePLND in prostate cancer are still open to debate, it provides accurate staging and remains the accepted and most effective method for detecting LN metastases and is the gold standard for assessing nodal status in prostate cancer [7-10]. The decision to extend the operation to remove nodes must be dictated by reasonable grounds. In our study, we aimed to present a combined technique using both an extraperitoneal and transperitoneal approach for ePLND, and confirm that such access can be safe for the patient and ergonomic for the surgeon.

Several publications have suggested that the extent and quality of a pelvic LND are not dependent on a specific surgical technique. Rather, nodal yield is far more dependent on surgeon's intent than the technical approach (open, laparoscopic, or robotic) [11]. At our center, all HR prostatectomies are performed by experienced operators. When deciding on a combination of LRP + ePLND, we always choose transperitoneal access, avoiding pelvic lymphocele, the most frequent complication after ePLND [12].

In 30 patients, we decided on a combined technique, which simultaneously used both access routes: a retroperitoneal access to remove the prostate and transperitoneal access for $L N$ removal. In all 30 patients, we first carried out prostatectomy using pre-peritoneal access in the conventional manner; we then moved the trocars into the peritoneum for lymphadenectomy. The movement of trocars into the peritoneal cavity was a very simple maneuver, taking up to 1 minute. During prostate removal, we paid special attention to the 13 patients in whom mpMRI indicated infiltration of the capsule. In the final histopathological examination, the presence of positive margins was confirmed in only 5 (16\%) patients versus $35 \%$ (range: $12-53 \%$ ) described in the literature [13]. Preservation of oncological purity by avoiding positive margins is a key condition for successful surgery. This is particularly difficult in HR patients with locally advanced cancer.

Combined technique also required modifying the position of the drains. We left a drain in the retroperitoneal space (in place of the left lower trocar) after completing the first stage of prostatectomy. The drain remained open throughout the course of the second stage, lymphadenectomy. There were exceptions to this practice in the case of 2 patients 
due to communication between spaces; in these patients, the drain remained closed. On the right side of these patients, we introduced an additional trocar with a $5 \mathrm{~mm}$ traction tool to lift the bladder. The second stage began by repositioning the first trocar for optics to the peritoneal cavity. This maneuver was easy because the peritoneum was always exposed during the creation of the retroperitoneal space. The introduction of insufflation into the cavity caused the retroperitoneal space to fall; the subsequent stage involving lymphadenectomy did not differ from the classical procedure. The time required for ePLND ranged from 35 to 85 min (mean: $56 \mathrm{~min}$ ). In a previous study, Katz et al. estimated that the operative time for PLND was $47 \mathrm{~min}$ in cases of standard PLND and 66-86 min (mean: $72 \mathrm{~min}$ ) in cases of ePLND [14] Similar times were reported by other authors. Yuh et al. reported a time of 3045 min [15], whereas Davis et al. quoted a time of 36-50 min [16]. However, in the current literature, only a few authors have described application of the extended PLND; papers have been confined mostly to limited or standard lymph node dissection (LND) [13]. During the removal of nodes, we always used the same scheme, beginning with dissection of the presacral lymphatics. Separate packets were sent from the perisacral, bilateral common iliacs, external iliacs, internal iliacs, and obturator. Heidenreich et al. suggested that it is unnecessary to dissect the presacral and common iliac lymphatics, as only 3.1\% of patients have LN metastases in that region [17]. However, in the present study, we identified metastases in up to five presacral nodes in 2 patients.

After removal of the presacral nodes, we moved along the common iliac artery, separating the ureter, and then along the external iliac artery to the node of Cloquet, which forms the upper boundaries of the preparation. The external border was the genitofemoral nerve. Initially, we were concerned that after the first retroperitoneal stage of prostate removal it would be difficult to carry out transperitoneal access to the presacral nodes and reach the triangle of Marcille [18]. However, we did not experience any difference between the classic and mixed operations except in two patients with pre-peritoneal and transperitoneal communication. We then used the traction tool introduced by the additional trocar to raise the bladder. Avoiding disturbance of the peritoneum during the first stage appears to be a key element of this procedure. This is not difficult, and we managed to carry it out successfully in 28 patients. The border for tissue removal along the internal iliac artery was the lateral sacral artery.

In a previous study, Jung et al. found that $25 \%$ of metastatic LNs are located in the internal iliac and common iliac regions [19]. The internal iliac nodes may be a primary landing zone, as 3 of 4 patients had exclusively internal iliac node metastasis [20]. This was also confirmed by our studies in which the most common location of metastases was the area of the internal iliac veins ( 11 positive and 8 subsequently positive obturator nodes). However, Mattei et al. reported that most LNs (38\%) were located alongside the external iliac and obturator packets [21]. These authors also found that $25 \%$ of primary lymphatic drainage occurs via the hypogastric nodes and $16 \%$ via the common iliacs vessels

All treatments involving our current patients were standard, and blood loss ranged from 200 to $400 \mathrm{ml}$. There was no need for intraoperative blood transfusion in any of our patients. Similar results have been published previously: $200 \mathrm{ml}(150-800$ $\mathrm{ml}$ ) [22] and $300 \mathrm{ml}$ (150-400 ml) [22]. However, in the present literature, only a few authors have actually published the use of ePLND rather than standard $\operatorname{LND}[13,23]$. According to the Clavien-Dindo classification, we observed grade 3 complications in three patients. In one case, we decided to re-operate.

One stage of RP for HR prostate cancer is entire prostate gland removal, which is done under operator-friendly conditions. The procedure is carried out in the retroperitoneal space with high $\mathrm{CO}_{2}$ pressure, which helps in hemostasis, with no pushing of the bowels, offering a good working space. In addition, performing anastomosis in the retroperitoneal space results in excellent visualization. The second stage of RP, ePLND, is much more feasible when performed via the transperitoneal approach. In the traditional technique, the very last part of RP (anastomosis) is the most difficult. During our combined technique, this stage is performed at the end of the first part of the operation (in the middle of the whole of procedure), when the surgeon is not as tired as at the end of long-lasting HR prostate cancer RP. This gives the opportunity to focus on meticulous anastomosis, which offers good functional results.

The main limitation of the present study is the small number of cases and short observation period. However, our encouraging results confirm the potential of combined techniques in the future. 


\section{Conclusions}

The combined technique is both feasible and safe. Good oncological purity, as measured by the number of negative margins and functional results, confirms the validity of the application of our combined technique. Performing the most difficult maneuver in HR prostate cancer (removal of the prostate in the first stage) appears to be more convenient for the operator. The timing of ePLND stage in the combined technique and the number of removed LNs do not differ from the standard lenticular access. Further comparative testing is necessary to demonstrate the clinical advantages of this new procedure.

\section{Conflict of interest}

The authors declare no conflict of interest.

\section{References}

1. Torre LA, Bray F, Siegel RL, et al. Global cancer statistics. CA Cancer J Clin 2015; 65: 87-108.

2. Cooperberg MR, Lubeck DP, Mehta SS, et al. CaPSURE. Time trends in clinical risk stratification for prostate cancer: implications for outcomes. J Urol 2003; 170: 21-5.

3. Van Poppel H, Joniau S. An analysis of radical prostatectomy in advanced stage and high-grade prostate cancer. Eur Urol 2008; 53: 253-9.

4. Spahn M, Joniau S, Gontero P, et al. Outcome predictors of radical prostatectomy in patients with prostate-specific antigen greater than $20 \mathrm{ng} / \mathrm{ml}$ : a European multi-institutional study of 712 patients. Eur Urol 2010; 58: 1-7.

5. Kim JK, Jeong CW, Ku JH, et al. Prostate specific antigen (PSA) persistence 6 weeks after radical prostatectomy and pelvic lymph node dissection as predictive factor of radiographic progression in node-positive prostate cancer patients. J Cancer 2019; 10: 2237-42.

6. Donohue JF, Bianco Jr FJ, Kuroiwa K, et al. Poorly differentiated prostate cancer treated with radical prostatectomy: long-term outcome and incidence of pathological downgrading. J Urol 2006; 176: 991-5.

7. Heidenreich A, Bellmunt J, Bolla M, et al.; European Association of Urology. EAU guidelines on prostate cancer. Part 1: screening, diagnosis, and treatment of clinically localised disease. Eur Urol 2011; 59: 61-71.

8. Hsu CY, Joniau S, Van Poppel H. Radical prostatectomy for locally advanced prostate cancer: technical aspects of radical prostatectomy. EAU Update Series 2005; 3: 90-7.

9. Ploussard G, Briganti A, de la Taille A, et al. Pelvic lymph node dissection during robot-assisted radical prostatectomy: efficacy, limitations, and complications - a systematic review of the literature. Eur Urol 2014; 65: 7-16.

10. Briganti A, Larcher A, Abdollah F, et al. Updated nomogram predicting lymph node invasion in patients with prostate cancer undergoing extended pelvic lymph node dissection: the essen- tial importance of percentage of positive cores. Eur Urol 2012; 61: 480-7.

11. Silberstein JL, Vickers AJ, Power NE, et al. Pelvic lymph node dissection for patients with elevated risk of lymph node invasion during radical prostatectomy: comparison of open, laparoscopic and robot-assisted procedures. J Endourol 2012; 26: 748-53.

12. Clark T, Parekh DJ, Cookson MS, et al. Randomized prospective evaluation of extended versus limited lymph node dissection in patients with clinically localized prostate cancer. J Urol 2003; 169: 145-7.

13. Yuh B, Artibani W, Heidenreich A, et al. The role of robot-assisted radical prostatectomy and pelvic lymph node dissection in the management of high-risk prostate cancer: a systematic review. Eur Urol 2014; 65: 918-27.

14. Katz DJ, Yee DS, Godoy G, et al. Lymph node dissection during robotic-assisted laparoscopic prostatectomy: comparison of lymph node yield and clinical outcomes when including common iliac nodes with standard template dissection. BJU Int 2010; 106: 391-6.

15. Yuh BE, Ruel NH, Mejia R, et al. Standardized comparison of robot-assisted limited and extended pelvic lymphadenectomy for prostate cancer. BJU Int 2013; 112: 81-8.

16. Davis JW, Shah JB, Achim M. Robot-assisted extended pelvic lymph node dissection (PLND) at the time of radical prostatectomy (RP): a video-based illustration of technique, results, and unmet patient selection needs. BJU Int 2011; 108: 993-8.

17. Heidenreich A, Varga Z, Von Knobloch R. Extendedpelvic lymphadenectomy in patients undergoing radical prostatectomy: high incidence of lymph node metastasis. J Urol 2002; 167: 1681-6.

18. Lee P, Francis KE, Solomon MJ, et al. Triangle of Marcille: the anatomical gateway to lateral pelvic exenteration. ANZ I Surg 2017; 87: 582-6.

19. Jung JH, Seo JW, Lim MS, et al. Extended pelvic lymph node dissection including internal iliac packet should be performed during robot-assisted laparoscopic radical prostatectomy for high-risk prostate cancer. I Laparoendosc Adv Surg Tech A 2012; 22: 785-90.

20. Musch M, Klevecka V, Roggenbuck U, et al. Complications of pelvic lymphadenectomy in 1,380 patients undergoing radical retropubic prostatectomy between 1993 and 2006. J Urol 2008; 179: 923-8

21. Mattei A, Fuechsel FG, Bhatta Dhar N, et al. The template of the primary lymphatic landing sites of the prostate should be revisited: results of a multimodality mapping study. Eur Urol 2008; 53: 118-25.

22. Yuh BE, Ruel NH, Mejia R, et al. Standardized comparison of robot-assisted limited and extended pelvic lymphadenectomy for prostate cancer. BJU Int 2013; 112: 81-8.

23. Dobruch J, Piotrowicz S, Skrzypczyk M, et al. Clinical value of extended pelvic lymph node dissection in patients subjected to radical prostatectomy. Videosurgery Miniinv 2014; 9: 64-70.

Received: 14.03.2019, accepted: 23.06.2019. 